\title{
Recent advances on the interval distance geometry problem
}

\author{
Douglas S. Gonçalves ${ }^{1}$ - Antonio Mucherino ${ }^{2}$ - Carlile Lavor ${ }^{3}$. \\ Leo Liberti ${ }^{4}$ (D)
}

Received: 28 January 2016 / Accepted: 20 December 2016

(C) Springer Science+Business Media New York 2016

liberti@lix.polytechnique.fr

Douglas S. Gonçalves

douglas.goncalves@ufsc.br

Antonio Mucherino

antonio.mucherino@irisa.fr

Carlile Lavor

clavor@ime.unicamp.br

1 CFM, Universidade Federal de Santa Catarina, Florianópolis, Brazil

2 IRISA, Université de Rennes 1, Rennes, France

3 IMECC, Universidade Estadual de Campinas, Campinas, Brazil

4 CNRS LIX, École Polytechnique, 91128 Palaiseau, France 


\section{Introduction}

Given a positive integer $K$ and a simple weighted undirected graph $G=(V, E, d)$, where $d$ maps edges $\{u, v\} \in E$ to positive interval weights $[\underline{d}(\{u, v\}), \bar{d}(\{u, v\})]$, the Distance Geometry Problem (DGP) [38] is the problem of finding a realization of the graph $G$ in a $K$-dimensional Euclidean space. In other words, the DGP requires the identification of a map $x: V \rightarrow \mathbb{R}^{K}$, satisfying the distance constraints:

$$
\underline{d}(\{u, v\}) \leq\|x(u)-x(v)\| \leq \bar{d}(\{u, v\}), \quad \forall\{u, v\} \in E,
$$

where $\|\cdot\|$ denotes the Euclidean norm.

A solution for (1) is called a realization or an embedding. In order to simplify the notation, we will use $x_{u}:=x(u)$ and $d_{u v}:=d(u, v):=d(\{u, v\})$ hereafter.

In structural biology, the problem of identifying molecular conformations from a given list of distance restraints between atom pairs is a DGP in dimension $K=3$. This problem is also known in the scientific literature as the Molecular Distance Geometry Problem (MDGP). In this particular application, the distances may be exact (i.e. $\underline{d}_{u v}=\bar{d}_{u v}$ ) or represented by a positive real-valued interval (i.e. $\bar{d}_{u v}>\underline{d}_{u v}>0$ ).

Exact distances are related to the chemical bonds whereas interval ones can be provided by experimental techniques. Such techniques include Nuclear Magnetic Resonance (NMR) [3], Förster resonance energy transfer (FRET) [7] and mass spectrometry (MS) cross-linking [10].

The DGP is NP-hard [56] and there exist several approaches to this problem (see [38, 52] and Sect. 1.1), where the DGP is reformulated as a global optimization problem on a continuous search domain, whose objective function is generally a penalty function of the distance constraints. More recently, a discrete approach to the DGP was proposed [39], where the continuous domain of the optimization problem is transformed into a discrete domain.

\subsection{Literature review}

Distance Geometry (DG) has played a prominent part in Global Optimization (GO) insofar as it has important applications to science (e.g. protein conformation) and engineering (localization of sensor networks, structural rigidity, control of unmanned underwater vehicles and robotic arms), and it is naturally cast as a system of nonconvex constraints (Eq. (1)) in terms of continuous decision variables. In general, DGPs are reformulated as a minimization of constraint violations. Such reformulations have the property that the optimal objective function value is zero for feasible instances, and strictly positive for infeasible ones. Various approaches have been proposed in this journal for the general case $[15,16,26,27,34,37,47,64,68]$, and many others on the application to protein conformation $[11,17,21,43,46,54]$. In this paper we focus on the case where the input graph is rigid, which implies that the search process has an inherently combinatorial side.

Over the years, the solution to MDGPs (DGPs arising in structural biology) have been typically attempted by employing tools such as ARIA [42], CYANA [23] and UNIO [22], which are all based on the Simulated Annealing (SA) meta-heuristic [28].

While molecular conformations are generally obtained by the above methods and successively stored in databases such as the Protein Data Bank (PDB) [4], a second class of methods based on Nonlinear Programming (NLP) solution techniques has emerged in the last decades. A well-known example is the DGSOL algorithm [48], which employs a homotopy method based on locally solving progressively finer Gaussian smoothings of the original problem. 
A third class of methods is based on Euclidean Distance Matrix Completion [1,14]. This is the case for the EMBED algorithm [12], which aims to fill in the missing distance bounds by constraint propagation of triangle and tetrangle inequalities. Thereafter, a candidate distance matrix (named dissimilarity matrix) is sampled from the completed interval distance matrix, and atom coordinates are obtained by matrix decomposition $[13,58]$. Since the dissimilarity matrix is not guaranteed to be a Euclidean Distance matrix, some of the original constraints might be violated. The last phase therefore consists in minimizing the constraint violation by local minimization, using the obtained embedding as an initial point.

A fourth class is centered around the so-called Build-Up algorithm [15,64]. These methods are based on the ancient idea of triangulation, used by humankind ever since navigation existed. In the context of distance geometry, where a point position is determined by the distances to it rather than the angles they subtend, this is known as "trilateration". Build-Up algorithms in dimension $K=3$ attempt to place an unknown point $x_{i}$ (for some $i \leq n$ ) by identifying at least four other points with known positions, and having known distances to $x_{i}$. When dealing with proteins and experimental data, the assumption of having four known exact distances to any given point may be excessively strong [44]. We point out, however, that some variants of the Build-Up algorithm overcome this limitation. For example, in order to address the uncertainty of the given distance values, the extension presented in [60] takes into account atomic coordinates and an unknown radius representing the uncertainty. Another variant [65] partly addresses the requirement of unknown vertices having at least four adjacent vertices with known positions. This variant can find multiple valid realizations, but appears to lack the ability to finding all possible incongruent realizations.

A fifth and very important class of methods is based on solving a Semidefinite Programming (SDP) relaxation of the DGP $[6,29,45]$. In particular, [29] exploits the cliques in the graph to reduce the size of the SDP formulation (also see [2]). This method was shown to be able to solve NMR instances containing real data and to reconstruct conformation models that are very close to the ones available on the PDB.

The authors of this paper are among the researchers who proposed and worked on a sixth class of methods based on a combinatorial algorithm called Branch \& Prune (BP) [36]. Protein graphs share some common properties: for example, they can be decomposed into a backbone subgraph and many side chains subgraphs [57] (these can be realized separately and then put together [55]). The backbone subgraph is larger than the subgraphs related to side chains, and hence most difficult to realize. However, it also defines an order on the atoms with certain topological properties, which we formally discuss below (informally, we can say that every atom in this order has at least three predecessors which are also adjacent in the graph structure). Under this assumption, the search domain of the underlying optimization problem can be reduced to a discrete set with a tree structure [32,51], which can be searched by the BP algorithm [36]. If the distances are exact, BP can find all realizations of a given protein backbone graph. Although an exhaustive search in the conformation tree is worst case exponential [32], numerical experiments have shown that BP behaves polynomially in protein-like instances [40]. In fact, it can be proved that, in such cases, the problem is Fixed Parameter Tractable (FPT) [41]. In computational experiments, the parameter value could always be fixed at a single constant, which explains the polytime behaviour. For protein backbone instances with exact distances, BP is one of the fastest available methods, one of the most reliable, and the only one which can certifiably find all incongruent realizations.

An adaptation of the BP to the interval distance setting was proposed in [34], where intervals are replaced with a finite set of discrete points. We refer to this BP adaptation as the interval BP $(i \mathrm{BP})$. This algorithm was tested on real protein instances in [8]. Although 
this BP variant shows promise, its practical applicability is currently limited by the choice of discretization points.

Several other approaches for solving DGPs can be found in the scientific literature. The interested reader can refer to $[33,38,52]$.

\subsection{Aim of this paper}

Our main motivation in this work is to improve the $i \mathrm{BP}$ algorithm proposed in [34] for solving MDGPs with interval data. For this purpose, we identify the main limitations of this discrete approach in presence of interval distances and propose a new variant of $i$ BP to find approximate solutions for interval MDGPs.

The identification of the barriers against the successful application of $i \mathrm{BP}$ in real settings represents an important step towards a combinatorial methodology with the following properties:

- it is specifically suitable for solving the protein conformation problem from distance restraint data;

- it can work with uncertain data, specified as interval distances provided by experimental techniques;

- it can potentially find all incongruent realizations of a given instance.

The paper is organized as follows. In Sect. 2, we define the subclass of DGP instances describing protein backbone graphs: we discuss assumptions, discretization orders, the $i \mathrm{BP}$ algorithm variant, pruning devices, and the parameterization of the coordinates. Section 3 presents a method, based on some interval distance constraints, which is able to reduce the set of candidate positions for certain vertices before the $i \mathrm{BP}$ branching phase. Section 4 addresses the main limitations in handling larger molecules in presence of interval data and presents a heuristic for finding approximate realizations. The computational results in Sect. 5 illustrate the improvements due to the proposed approaches.

\section{A combinatorial approach}

Let $G=(V, E, d)$ be a simple weighted undirected graph representing an instance of the MDGP. In the following, vertices of $V$ will represent atoms of the given molecule and $\{u, v\} \in$ $E$ if the distance between the atoms $u$ and $v$ is available. The map $d$ relates each edge $\{u, v\} \in$ $E$ to a positive interval weight $[d(u, v), \bar{d}(u, v)]$. The MDGP asks to find a realization $x: V \rightarrow \mathbb{R}^{3}$ (see Introduction), i.e. a molecular conformation in three-dimensional space such that:

$$
\underline{d}(u, v) \leq\left\|x_{u}-x_{v}\right\| \leq \bar{d}(u, v), \quad \forall\{u, v\} \in E .
$$

Recall that $\underline{d}(u, v)$ and $\bar{d}(u, v)$ denote, respectively, the lower and upper bounds for the distance $d(u, v)$ (with $\underline{d}(u, v)=\bar{d}(u, v)$ if $d(u, v)$ is exact). We also suppose that the given set of distances is realizable in $\mathbb{R}^{3}$.

In order to discretize the search domain, MDGP instances need to satisfy some particular assumptions. The main requirement is that the atoms need to be sorted in a way that there are at least three reference atoms for each of them (aside, obviously, from the first three). We say that an atom $u$ is a reference for another atom $v$ when $u$ precedes $v$ in the given atomic order, and the distance $d(u, v)$ is known. In such a case, candidate positions for $v$ belong to the sphere centered in $u$ and having radius $d(u, v)$. When the reference distance $d(u, v)$ is given through an interval, the sphere becomes a spherical shell, namely, the region between 
Fig. 1 The two feasible arcs obtained by intersecting two spheres and one spherical shell

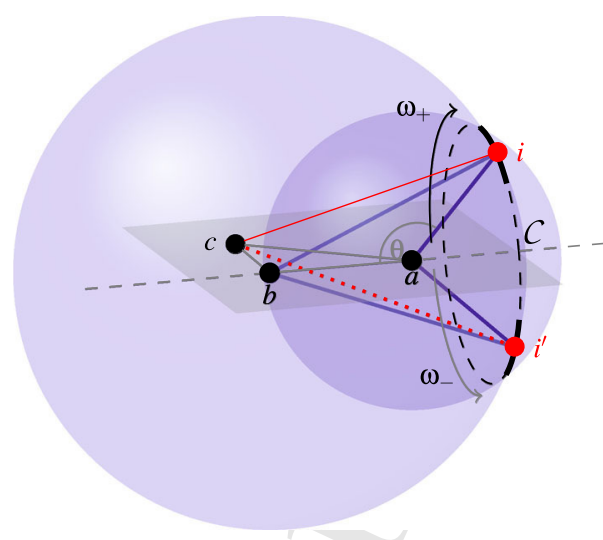

an inner sphere of radius $d$ and an outer sphere of radius $\bar{d}$ with the same center. If three reference atoms are available for $v$, then candidate positions (for $v$ ) belong to the intersection of three spherical shells. The easiest situation is the one where the three available distances are exact and the intersection gives, in general, two possible positions for $v$ [32]. However, if only one of the three distances is allowed to take values into a certain interval, then the intersection gives two arcs of a circle, generally disjoint, where sample points can be chosen [34]. In both last situations, the discretization can be performed. More details are given in the next section.

\section{1 $i \mathrm{BP}$ algorithm and discretization orders}

Let $G=(V, E, d)$ be an instance of the MDGP and let us suppose that there exist an order for the atoms $v \in V$, so that we can assign a numerical label $i \in\{1,2, \ldots,|V|\}$ to each of them. At each recursive call of the $i \mathrm{BP}$ algorithm, candidate positions for the current atom $i$ are computed using the positions of the previously placed reference atoms and their distances to the atom $i$.

When the distances between $i$ and its references are exact, the intersection of three spheres needs to be computed. If the reference atoms $\{a, b, c\}$ are not collinear, then such an intersection results in at most two points. When this situation is verified for all atoms $i>3$, then the search domain has the structure of a binary tree [32].

However, if one of the three reference distances, say $d_{c i}$, is an interval, then the two spheres centered at $x_{a}$ and $x_{b}$ need to be intersected with a spherical shell centered at $x_{c}$. As a result, the intersection gives two candidate arcs (see Fig. 1). These arcs are over the dashed circle $\mathcal{C}$ defined by the intersection of the two spheres. When the intersection consists of two arcs, a finite number $D$ of sample positions should be selected from each of them [34]. This way, we still have a discrete set of possible positions for the atom $i$.

Therefore, the discretization strongly depends on an order for the vertices (atoms) of $G$ satisfying specific properties. Definition 1 formalizes the assumptions mentioned above.

Definition 1 The interval Discretizable DGP in dimension $3\left(i \mathrm{DDGP}_{3}\right)$

Given a simple weighted undirected graph $G=(V, E, d)$, where $E^{\prime} \subset E$ is the subset of edges for which their weights are exact distances, we say that $G$ represents an instance of the $i$ DDGP $_{3}$ if there exists a total order on the vertices of $V$ verifying the following conditions:

(a) $G_{C}=\left(V_{C}, E_{C}\right) \equiv G[\{1,2,3\}]$ is a clique and $E_{C} \subset E^{\prime}$;

(b) $\forall i \in\{4, \ldots,|V|\}$, there exists $\{a, b, c\}$ such that 


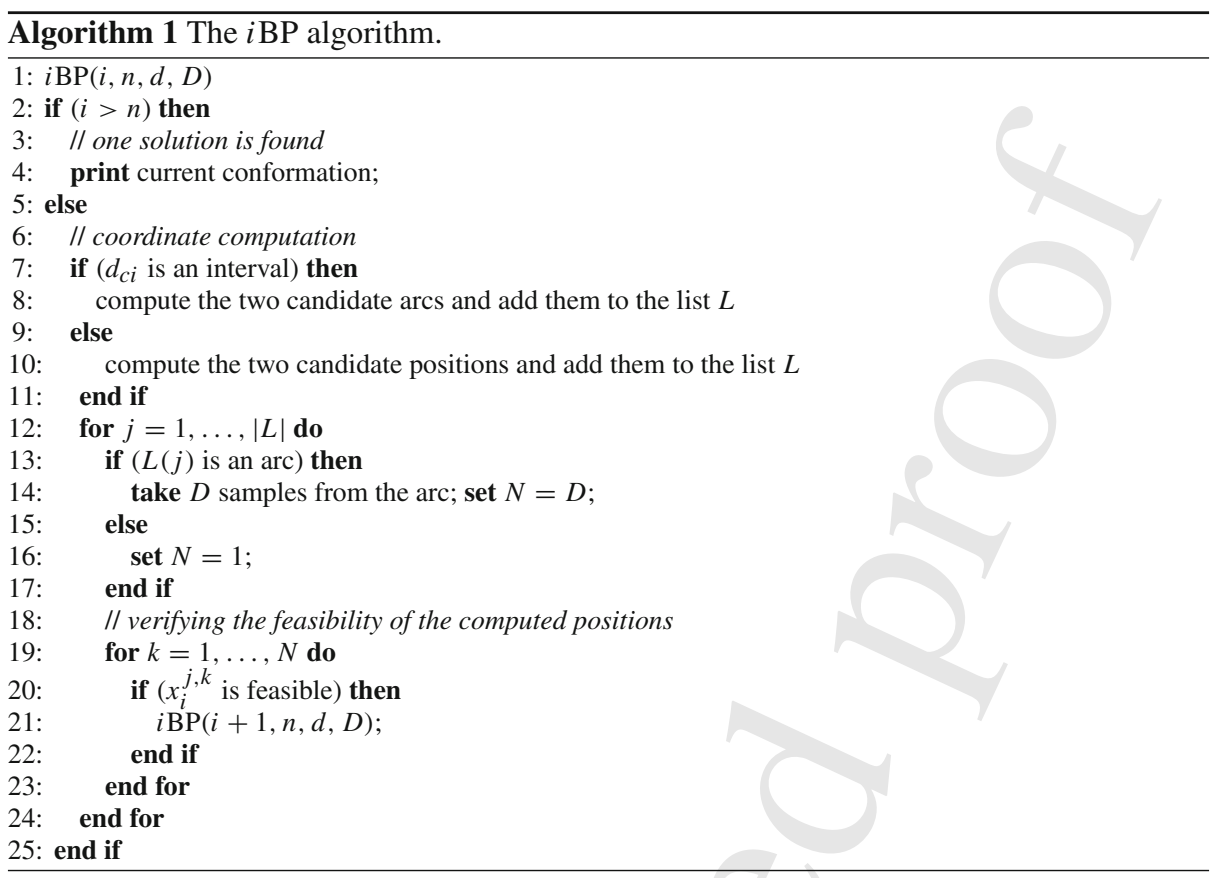

1. $a<i, b<i, c<i$;

2. $\{\{b, i\},\{c, i\}\} \subset E^{\prime}$ and $\{a, i\} \in E$;

3. $\Delta_{S}(a, b, c)>0$,

where $\Delta_{S}(a, b, c)$ stands for the area of the triangle formed by $\{a, b, c\}$. Assumption (a) allows us to place the first 3 atoms uniquely and fixes the realization with respect to rotation and translations. Assumptions (b.1) ensures the existence of three reference atoms for every $i>3$, and assumption (b.2) ensures that at most one of the three reference distances may be represented by an interval. Finally, assumption (b.3) requires that the area $\Delta_{S}(a, b, c)$ is strictly positive, which prevents the references from being collinear. Under these assumptions, the MDGP can be discretized.

Algorithm 1 is a sketch of the $i \mathrm{BP}$ algorithm for solving $i \mathrm{DDGP}_{3}$ instances. In the algorithm call, $i$ is the current atom for which the candidate positions are searched, $n$ is the total number of atoms forming the considered molecule, $d$ is the list of available distances (exact or interval distances), and $D$ is the discretization factor, i.e. the number of sample points that are taken from the arcs in case the distance $d_{c i}$ is represented by an interval. In the algorithm (see lines 8 and 10), we make use of a list $L$ of positions and arcs, from which candidate positions are extracted.

Given an order for the vertices in $V$ satisfying the assumptions in Definition 1, the algorithm calls itself recursively in order to explore the tree of candidate positions. Every time a new atomic position is computed, it defines a new branch of the tree. This phase in $i \mathrm{BP}$ is named branching phase. For every computed atomic position, its feasibility is verified by checking the constraints (2), up to the current tree layer, or other additional feasibility criteria based on properties of the molecule, e.g, van der Waals' separation distance (VdW), chirality constraints, and others $[8,53]$. This phase in $i \mathrm{BP}$ is named pruning phase, and the criteria are called pruning devices (see line 20 of Algorithm 1). 


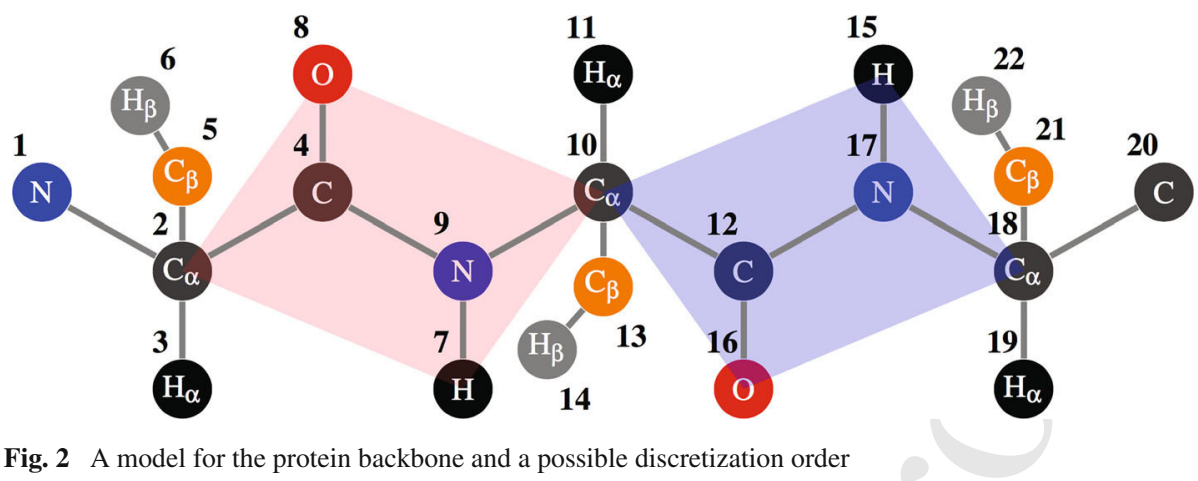

Even if the tree grows exponentially (in the worst case scenario), the pruning devices allow $i \mathrm{BP}$ to focus the search on the feasible parts of the tree. The easiest and most efficient pruning device is the Direct Distance Feasibility (DDF) criterion [32], which consists in verifying the $\epsilon$-feasibility of constraints involving distances between the current atom $i$ and previously placed atoms:

$$
\underline{d}(h, i)-\epsilon \leq\left\|x_{h}-x_{i}\right\| \leq \bar{d}(h, i)+\epsilon, \quad \forall\{h, i\} \in E, \text { with } h<i \text { and } h \notin\{a, b, c\} .
$$

The distances involved in the above constraints are called pruning distances.

\subsection{Protein backbone model: discretization orders and pruning devices}

A necessary preprocessing step for solving MDGPs by this discrete approach consists in finding suitable atomic orders allowing each atom $v$ to have at least three reference atoms. We name such orders discretization orders [9]. In previous works, discretization orders have been either handcrafted [34], or obtained by looking for paths on pseudo de Bruijn graphs consisting of cliques of $G$ [50], or even automatically detected by a greedy algorithm [31,49]. In fact, if we consider distances defined by bond lengths and bond angles as exact, along with the peptide plane geometry, it is possible to find orders for the protein backbone (and also for side chains [11]) satisfying the assumptions required for the discretization. This preprocessing step can be performed efficiently, in polynomial time [49], so that the necessary assumptions can be fulfilled by graphs related to proteins. However, we point out that when some additional assumptions are imposed to the searched orders, such as the consecutivity of the reference vertices, this problem becomes NP-hard [9]. In this work, we consider a model for the protein backbone as depicted in Fig. 2.

With the backbone atoms $\mathrm{N}, \mathrm{C}_{\alpha}$, and $\mathrm{C}$, we also considered the attached $\mathrm{H}$ and $\mathrm{H}_{\alpha}$, and we have included only the $\mathrm{C}_{\beta}$ and $\mathrm{H}_{\beta}$ atoms to represent the side chain of each amino acid. There are two exceptions for this amino acid model: the glycine, where $\mathrm{C}_{\beta}$ and $\mathrm{H}_{\beta}$ are replaced by one $\mathrm{H}$, and the proline, which has a missing hydrogen. The backbone model in Fig. 2 only considers 3 amino-acids, but it can be repeated for all amino-acids in a longer protein sequence, because of the regular pattern defining the protein backbone.

The order depicted in Fig. 2 is the one used in our numerical experiments.

The first three atoms, $\mathrm{N}-\mathrm{C}_{\alpha}-\mathrm{H}_{\alpha}$, of the first amino-acid can be used as initial clique (see assumption (a) in Definition 1) for the discretization order because the involved distances are defined by bond lengths and angles, that can be considered as exact [12]. Analogously, taking into account the peptide plane distances and the distances between hydrogens provided by 
NMR, it is not hard to verify that assumptions (b.1)-(b.2) of Definition 1 are satisfied by the order given in Fig. 2.

On the basis of the model in Fig. 2 for the protein backbone geometry, it is possible to conceive other pruning devices $[8,53]$ to be integrated with DDF (see, Eq. 3), based on the following considerations:

- Helices in proteins can be either right or left-handed. The former situation is statistically more common, because of side chains steric constraints. In this work, we do not consider side chains explicitly, but we suppose that it is possible to understand, from an analysis of the protein sequence, whether right-handed or left-handed helices are expected to be present. We call this pruning device as the chirality-based device: in some situations, it can allow for placing uniquely some atoms during the execution of the search. For the carbon $\mathrm{C}$, in fact, we can get only one (instead of two) possible positions by using $\mathrm{N}-\mathrm{C}_{\alpha}-\mathrm{H}_{\alpha}$ as reference atoms. An analogous reasoning can be applied to $\mathrm{C}_{\beta}$. The chirality defines the orientation of the tetrahedron formed by $\mathrm{C}, \mathrm{C}_{\beta}, \mathrm{N}, \mathrm{C}_{\alpha}, \mathrm{H}_{\alpha}$, where $\mathrm{C}_{\alpha}$ is the chiral center, and it can be used to avoid unnecessary branching;

- The tetrahedron around $\mathrm{C}_{\alpha}$ forms a clique as well as the peptide planes [2]. Such local structures define rigid regions of the protein backbone. Using the peptide plane clique, it is possible to find a unique position for $\mathrm{C}_{\alpha}$. It is also possible to place $\mathrm{N}$ uniquely, because its relative orientation with respect to $\mathrm{H}, \mathrm{C}$ and $\mathrm{C}_{\alpha}$ of the same peptide plane can be computed by taking into account the van der Waals minimum distance;

- The oxygen atoms in Fig. 2 are included in the model because they participate in hydrogen bonds. Each oxygen can be placed uniquely by using the exact distances with the other atoms of the peptide plane.

\subsection{Computing coordinates for candidate positions}

The method employed to compute the candidate positions at each recursive call of Algorithm 1 has a fundamental importance. While looking for candidate atomic positions for the atom $i$, it is supposed that the reference atoms $\{a, b, c\}$ are already positioned. These reference atoms define a local coordinate system centered at $a[19,62]$. This coordinate system is illustrated in Fig. 3.

Let $v_{1}$ be the vector from $b$ to $a$ and $v_{2}$ be the vector from $b$ to $c$. The $x$-axis for the system in $a$ can be defined by $v_{1}$, and the unit vector in this direction is $\hat{x}=v_{1} /\left\|v_{1}\right\|$. Moreover, the vectorial product $v_{1} \times v_{2}$ gives another vector that defines the $z$-axis, whose corresponding unit vector is $\hat{z}$. Finally, the vectorial product $\hat{x} \times \hat{z}$ provides the vector that defines the $y$-axis (let the unit vector be $\hat{y}$ ).

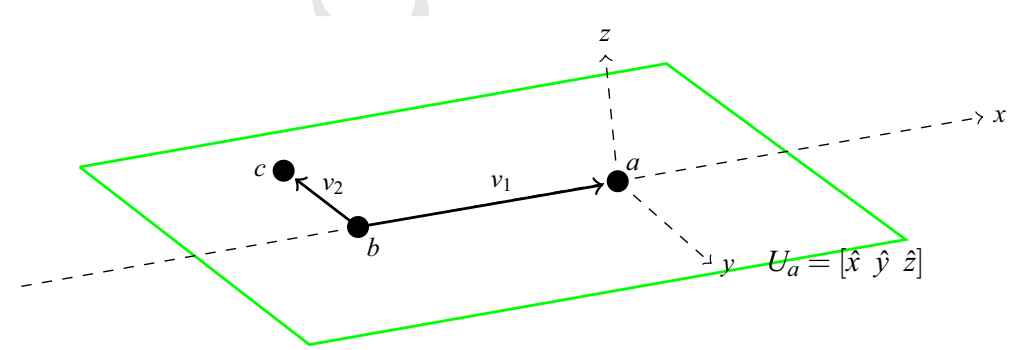

Fig. 3 The reference vertices $a, b$ and $c$ induce a system of coordinates 


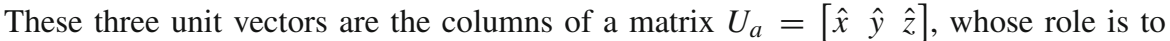
convert directly vertex positions from the coordinate system defined in $a$ to the canonical system.

Once the matrix $U_{a}$ has been computed, the canonical Cartesian coordinates for a candidate position for the vertex $i$ can be obtained by:

$$
x_{i}\left(\omega_{i}\right)=x_{a}+U_{a}\left[\begin{array}{r}
-d_{a i} \cos \theta_{i} \\
d_{a i} \sin \theta_{i} \cos \omega_{i} \\
d_{a i} \sin \theta_{i} \sin \omega_{i}
\end{array}\right],
$$

where $\theta_{i}$ and $\omega_{i}$ are the angles related to the spherical coordinates of vertex $i$.

We will use the symbol $\theta_{i}$ in order to refer to the angle formed by the two segments $(i, a)$ and $(a, b)$, and we will use the symbol $\omega_{i}$ to refer to the angle formed by the two planes defined by the triplets $(a, b, c)$ and $(b, a, i)$ (see Fig. 1). The cosine of the angles $\theta_{i}$ and $\omega_{i}$ can be computed by exploiting the positions of the reference vertices $a, b$ and $c$, as well as the available distances $d_{a i}, d_{b i}$ and $d_{c i}$. Thus,

$$
\cos \omega_{i}=\frac{\cos \theta_{c, b, i}-\cos \theta_{a, b, i} \cos \theta_{a, b, c}}{\sin \theta_{a, b, i} \sin \theta_{a, b, c}},
$$

where we consider the positive values for the sines, and

$$
\cos \theta_{i}=\cos \theta_{b, a, i}=\frac{d_{a b}^{2}+d_{a i}^{2}-d_{b i}^{2}}{2 d_{a b} d_{a i}} .
$$

Recall from Sect. 2.1 that if the three reference distances are all exact, then the three spherical shells are in fact three spheres, whose intersection gives 2 points, with probability 1 [32]. The two points $x_{i}^{+}$and $x_{i}^{-}$correspond to two possible opposite values, $\omega_{i}^{+}$and $\omega_{i}^{-}$, for the angle $\omega_{i}$. When one of the three distances is instead represented by an interval (see Definition 1), the third sphere becomes a spherical shell, and the intersection provides two curves (see Fig. 1). These two curves correspond to two intervals, $\left[\underline{\omega}_{i}^{+}, \bar{\omega}_{i}^{+}\right]$and $\left[\underline{\omega}_{i}^{-}, \bar{\omega}_{i}^{-}\right]$, for the angle $\omega_{i}$. In order to discretize these intervals, a certain number of points, say $D$, can be chosen from the two curves.

As shown in [19], the generalized procedure for the computation of atomic coordinates in Algorithm 1, based on equation (4), is very stable when working on MDGP instances related to real proteins. Moreover, equation (4) is also at the basis of an important technique that can be used to reduce the feasible arcs obtained by sphere intersection. This technique for arc reduction was firstly proposed in [20]. Another approach for arc reduction, based on Clifford algebra, is presented in [30].

\section{Pruning distances and arc reduction}

When candidate atomic positions, at each recursive call of the $i \mathrm{BP}$ algorithm (see Algorithm 1), are computed by intersecting two spheres with one spherical shell, a continuous set of positions is obtained, which generally corresponds to two disjoint arcs, related to two intervals for the corresponding torsion angle values.

During a typical run of Algorithm 1, every time the reference distance $d_{c i}$ is represented by an interval, $D$ equidistant samples are taken from each arc [34]. As a consequence, $2 D$ atomic positions are generated in total, and $2 \mathrm{D}$ new branches are added to the tree, at the current layer, for every branch at the upper level. After their computation, the feasibility of each atomic position is verified. On the one hand, too large $D$ values can drastically 
increase the width of the tree; on the other hand, too small values can generate trees where no solutions can be found (all branches are pruned, because all positions, at a certain layer, are not compatible with pruning distances).

In [20], an adaptive scheme was proposed for tailoring the branching phase of the $i \mathrm{BP}$ algorithm so that all computed candidate positions are feasible at the current layer. The idea is to identify, before the branching phase of the algorithm, the subset of positions on the two candidate arcs that is feasible with respect to all pruning distances to be verified on the current layer.

Let us suppose that, at the current layer $i$, the distance $d_{c i}$ is represented by the interval $\left[\underline{d}_{c i}, \bar{d}_{c i}\right]$. By using Equation (4), two intervals for the angle $\omega_{i}$ can be identified: $\left[\underline{\omega}_{i}^{+}, \bar{\omega}_{i}^{+}\right] \subset$ $[0, \pi]$ and $\left[\underline{\omega}_{i}^{-}, \bar{\omega}_{i}^{-}\right] \subset[\pi, 2 \pi]$, such that the distance constraints

$$
\begin{aligned}
\left\|x_{a}-x_{i}\left(\omega_{i}\right)\right\| & =d_{a i}, \\
\left\|x_{b}-x_{i}\left(\omega_{i}\right)\right\| & =d_{b i}, \\
\underline{d}_{c i} \leq\left\|x_{c}-x_{i}\left(\omega_{i}\right)\right\| & \leq \bar{d}_{c i},
\end{aligned}
$$

are satisfied.

However, there may be pruning distances, at layer $i$, that could be exploited for tightening these two arcs. Let us suppose there is an $h \in\{j<i \mid j \notin\{a, b, c\}\}$, such that the distance $d_{h i}$ is known and lies in the interval $\left[\underline{d}_{h i}, \bar{d}_{h i}\right]$. The solution set of the inequalities

$$
\underline{d}_{h i} \leq\left\|x_{h}-x_{i}\left(\omega_{i}\right)\right\| \leq \bar{d}_{h i}
$$

consists of intervals for $\omega_{i}$ that are compatible with the distance $d_{h i}$.

A discussion about how to solve the inequalities (6), by using Eq. (4), is presented in details in [20].

The feasible positions for the atom $i$ can be therefore obtained by intersecting the two previously computed arcs (in bold in Fig. 1), and several spherical shells, each of them defined by considering one pruning distance between $i$ and $h<i$. For each available pruning distance, other inequalities (6) can be defined and new arcs on the circle $\mathcal{C}$ may be identified. The final subset of $\mathcal{C}$ which is compatible with all available distances can be found by intersecting the arcs obtained for each pruning distance with the two initial disjoint arcs, given by Eq. (5).

After considering all pruning distances, i.e., after performing all intersections, the final result provides a list of $\operatorname{arcs}$ on $\mathcal{C}$ that are feasible with all the distances that can be verified at the current layer. All positions that can be taken from these arcs are feasible at the current layer: all of them generate a new branch and may serve as a reference for computing new candidate positions on deeper layers of the tree. In order to integrate the $i \mathrm{BP}$ algorithm with this adaptive scheme, there are two main changes to be performed on Algorithm 1. On line 8, the adaptive scheme needs to be invoked for taking into consideration the information about the pruning distances. Moreover, the use of the DDF pruning device has become unnecessary, and it should not be considered at line 20 of Algorithm 1.

It is important to remark that this adaptive scheme is not supposed to speed up the execution of the search, but rather to help in defining search trees that can actually contain solutions. Without the use of this adaptive scheme, all sample positions selected from the two arcs obtained with the discretization may be discovered to be infeasible as soon as the DDF pruning device is invoked. The other extreme situation is instead the one where the adaptive scheme can allow us to select a subset of sample positions that all bring to the definition of a solution. Naturally, the second situation is desirable, even if, in terms of complexity, it tends to increase the total computational cost. 
Fig. 4 Realization of five points in $\mathbb{R}^{3}$

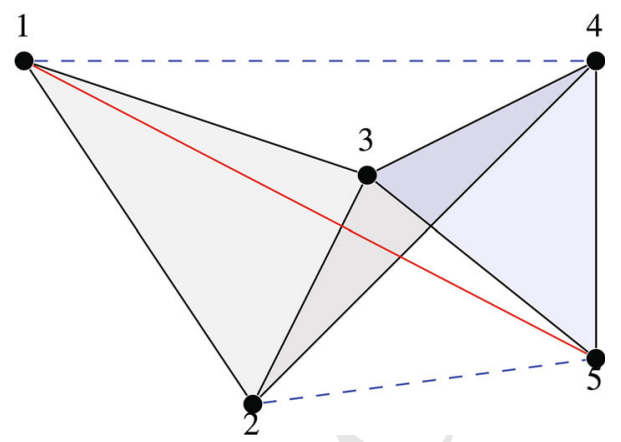

\section{Limitations of the current approach: finding approximate realizations}

For DGP instances where all available distances are exact, the presented discrete approach is extremely efficient, allowing for example to realize graphs having thousands of vertices in few seconds with a standard computer [32].

However, for $i$ DDGP $_{3}$ instances, there are some difficulties encountered by the $i \mathrm{BP}$ algorithm [34], even for finding one solution. Such limitations, related to the presence of interval data in both discretization and pruning distances, are discussed in this section and a heuristic to overcome such barriers is proposed.

\subsection{Sampled distances and embeddability}

Recent computational experiments have shown that taking equidistant sample points on the feasible arcs (or equidistant samples from the interval distance, see Algorithm 1 in Sect. 2.1), even after the intersection with the available pruning distances (see Sect. 3), is not enough to allow the $i \mathrm{BP}$ algorithm to solve some MDGPs within a predefined precision. The sampled distances are taken independently in each layer of the tree and, in particular for small $D$ values, it is not likely that they are compatible with each other and with other pruning distances available at deeper layers.

The underlying issue is related to the embeddability of a given set of distances. Suppose that we are positioning the atom $i$ and that the interval distance $\left[\underline{d}_{c i}, \bar{d}_{c i}\right]$ is used in the discretization. Even if we assume that there exists a distance value $d_{c i}^{*}$ which is compatible with the other distances in $E$ leading to a solution, we cannot ensure that, with a finite number $D$ of samples taken from $\left[\underline{d}_{c i}, \bar{d}_{c i}\right]$, the compatible distance $d_{c i}^{*}$ is actually sampled.

In order to illustrate this fact, consider the following example where five points in $\mathbb{R}^{3}$ are embedded (Fig. 4).

Suppose that the straight lines represent exact distances, and let the black lines be the exact distances used in the discretization. The dashed blue lines are the interval distances (used to compute the possible positions of atoms 4 and 5) and the red straight line represents one pruning distance (that can be used to validate the possible positions for the atom 5). The associated distances are the following: $d_{12}=d_{23}=d_{24}=d_{34}=d_{35}=d_{45}=1, d_{13}=\sqrt{2}$, $d_{14}=\sqrt{x} \in[0.5,2], d_{15}=\sqrt{3}, d_{25}=\sqrt{y} \in[0.5,2]$.

According to the Cayley-Menger conditions $[38,59]$, for this set of distances to be realizable in $\mathbb{R}^{3}$, it is necessary that 


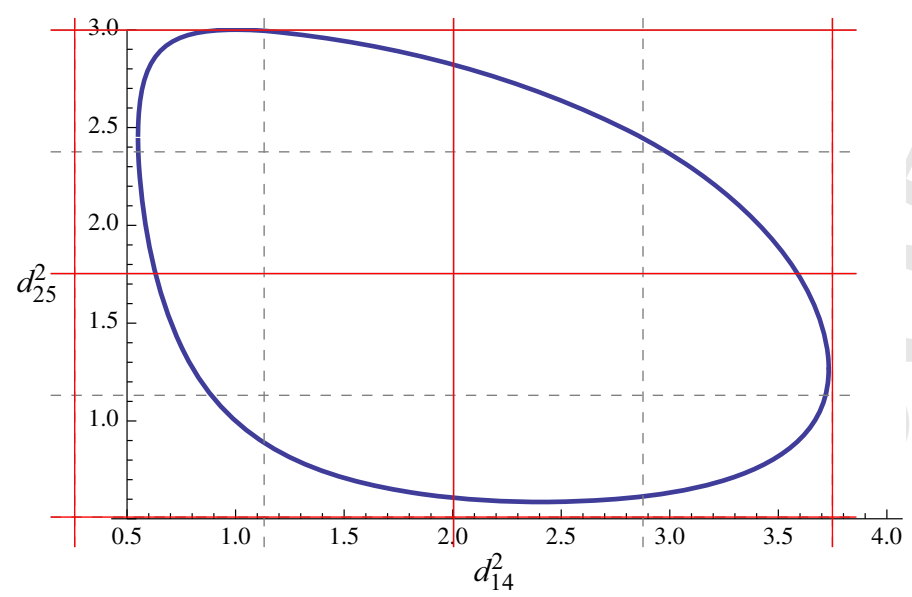

Fig. 5 Solution set for the five point Cayley-Menger determinant with $d_{14}^{2}$ and $d_{25}^{2}$ as missing distances

where the above matrix is a bordered distance matrix and $|\cdot|$ denotes its determinant. The solution set of this equation (the values for the missing (interval) squared distances $x=d_{14}^{2}$ and $y=d_{25}^{2}$ ) is represented by the blue curve in Fig. 5 .

It is easy to see that, unless the grid is sufficient refined (number of samples $D$ is sufficient large), a valid pair of distances $\left(d_{14}^{2}, d_{25}^{2}\right)$ can be sampled with probability zero.

\subsection{Long-range distance restraints}

Long-range distance restraints are related to atoms that are at least four amino-acids apart in the protein sequence. Even if far in the protein sequence, some atom pairs may be in condition to be detected by an experimental technique. For example, if we consider NMR, it is typical to detect distances between atoms that are very far in the sequence, but quite close in space $(\leq 5 \AA)$.

In case of all available distances are exact, the pruning distances efficiently guide the search in the binary tree corresponding to the discretized search space $[32,40]$. However, when interval distances are present, the search tree is no longer binary, because $D$ samples are taken from each feasible arc. Moreover, the DDF pruning criterion (3) becomes much less effective when the bounds $[\underline{d}, \bar{d}]$ are loose, resulting in a large number of active nodes in the tree, which increases exponentially the cost of exploring a whole subtree.

Furthermore, since other interval distances are also employed in the discretization, the sampled positions in the feasible arcs for previous atoms are only approximations for their true positions, and such a sequence of approximate positions may lead to an infeasibility at a further layer. For this reason, the longest-range pruning distances may fail to be verified (even if they are represented by an interval).

\section{를 Springer}



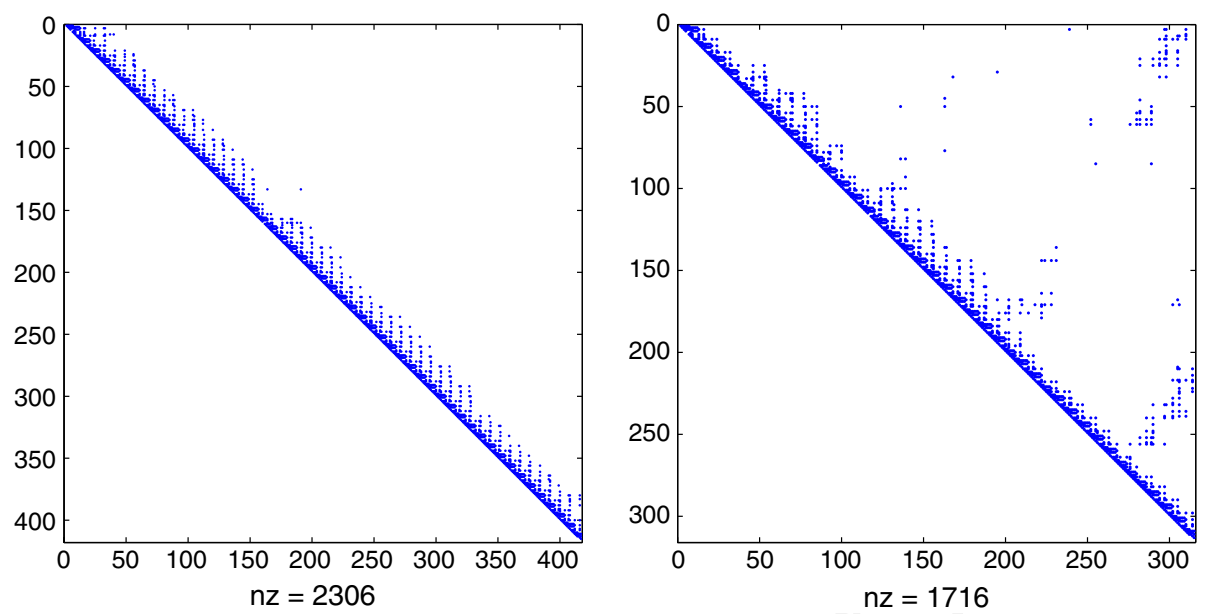

Fig. 6 Available distances for the instances 1FJK (left) and 2E2F (right)

To illustrate this fact, we depict in Fig. 6 the available entries of the upper triangular part of the distance matrices for two instances belonging to our set: $1 \mathrm{FJK}$ and $2 \mathrm{E} 2 \mathrm{~F}$. Notice that the almost-band structure close to the main diagonal is a consequence of the assumptions concerning the discretization. In fact, the distances between pairs $(i-1, i)$ and $(i-2, i)$ are generally derived from the bond lengths and angles, while the distances $(i-3, i)$ can be generally obtained from the analysis of the torsion angle among the quadruplet of atoms $(i-3, i-2, i-1, i)$. Moreover, the distance $(i-3, i)$ may be also estimated by applying an experimental technique such as NMR. Other distances that are far from the main diagonal of the matrix can be obtained applying an experimental technique.

Although 1FJK has more atoms than 2E2F, the former instance can be easily solved by $i$ BP in a few seconds, whereas the latter cannot be solved in less than one minute (using $D \leq 20$ ). The difficulty in solving $2 \mathrm{E} 2 \mathrm{~F}$ is related to the presence of long-range pruning distances: there are several entries in its distance matrix that are far from the diagonal.

\subsection{Approximate realizations}

The presence of interval distances implies uncertainty on the atomic positions obtained by sampling points in the intersection between spheres and spherical shells: even a small error introduced at tree layer $i$ can have a relevant propagation until the layer $j>>i$ and, when the pruning distance is finally tested, it is likely that the propagated error leads to infeasible positions for atom $j$.

Thus, an error introduced during the intersection discretization in a certain tree layer, might make every sampled candidate position infeasible with pruning distances in a further layer. This phenomenon is more evident when considering long-range distance restraints. One possibility to avoid pruning out all branches of the search tree, in order to obtain approximate solutions to the problem, is to relax the distance constraints related to long-range distances. We define the set

$$
\mathcal{L}=\{\{i, j\} \in E|| i-j \mid \geq M\},
$$


where $M$ is a positive integer used to identify long-range distance restraints. Our relaxation consists in avoiding the application of the DDF feasibility test (Eq. 3), as well as the intersection scheme (Sect. 3), to pruning distances in $\mathcal{L}$.

Naturally, when such pruning distances are neglected, some information is lost and this can have an impact on the found solutions. In fact, long-range distance restraints are the main responsible for the global fold. Thus, in order to mitigate this effect, we introduce another pruning criterion based on the partial Mean Distance Error (MDE) at the current layer $k$ :

$$
\operatorname{PMDE}_{k}(X)=\frac{1}{\left|J_{k}\right|} \sum_{\{i, j\} \in J_{k}}\left[\frac{\max \left\{\underline{d}_{i, j}-\left\|x_{i}-x_{j}\right\|, 0\right\}}{\underline{d}_{i, j}}+\frac{\max \left\{\left\|x_{i}-x_{j}\right\|-\bar{d}_{i, j}, 0\right\}}{\bar{d}_{i, j}}\right],
$$

where

$$
J_{k}=\{\{i, j\} \in E \mid i \leq k \wedge j \leq k\} .
$$

Let $n=|V|$ and note that $J_{n}=E$. It is common to measure the quality of a realization by the Mean Distance Error measure:

$$
\operatorname{MDE}(X)=P M D E_{n}(X) .
$$

Thus, by monitoring the $P M D E_{k}(X)$ for $k<n$, we can control the quality of partial realizations. This suggests the PMDE pruning device: if at layer $k, P M D E_{k}(X)>\hat{\varepsilon}$, then the candidate partial realization should be pruned. We set $\hat{\varepsilon}>\varepsilon$, where $\varepsilon$ is the tolerance used in DDF (Eq. 3).

When this new pruning device is introduced, a solution found by Algorithm 1 is actually an approximate solution in the sense that it satisfies all distances in $E \backslash \mathcal{L}$ (with tolerance $\varepsilon$ ), while some distances in $\mathcal{L}$ can be violated. However, the total MDE value for such a solution remains relatively small, because of the new pruning device based on (8). By applying this scheme, together with the chirality and peptide plane constraints (see Sect. 2.2), we expect that the fold of the obtained conformation mimics the fold of the true protein. This is the case for the set of instances used in the computational experiments.

\section{Computational experiments}

In this section we present some computational results on a set of artificially generated MDGP instances. Our aim is to assess the improvements on $i \mathrm{BP}$ (Algorithm 1) due to the integration of a set of recently proposed techniques: the pruning devices based on chirality and peptide plane geometry, described in Sect. 2.2; the arc reduction technique presented in Sect. 3; and the partial MDE pruning device introduced in Sect. 4.3.

The instances that we consider in our experiments were generated as it follows. The protein conformations were extracted from the PDB: by using the coordinates of a known conformation, all pairwise distances between atom pairs of the backbone were computed. Then, only a small subset of all distance pairs is kept for defining an instance. The distances related to bond lengths and those that can be obtained from bond angles are considered as exact, as well as distances between atoms belonging to the same peptide plane (see Fig 2). Torsion angles on the protein backbones give rise to the definition of interval distances, related to the minimal and maximal extension of such torsion angles. Distances between pairs of hydrogens are also included, as specified in the next subsection. 


\subsection{Assumptions concerning distances between hydrogens}

During the generation of our instances, we rely on the premise that experimental techniques, such as NMR spectroscopy, are able to give information about all distances between hydrogen atoms that are close in space [35]. Moreover, these distances can be supposed to be more precise than other ones. Statistics on such distances [5,66], with the geometry of consecutive peptide planes, validate this assumption.

We will consider therefore that all distances between hydrogens belonging to the same or to two consecutive amino-acids are available, and we suppose that they lie in an interval having width $0.1 \AA$. Besides these distances, responsible for the local geometry, we also consider distances between hydrogens that belong to amino acids that are far in the protein sequence, but close in space. These distances are responsible for the global fold. We include these distances in our generated instances whenever they are smaller than $5 \AA$ and consider that imprecisions lead to an interval of width $1 \AA$.

Hydrogen bonds $H-O$, responsible for stabilizing $\alpha$-helices and $\beta$-strands, are also considered. If the distance between $H$ and $O$ of distinct amino-acids is greater than $1.3 \AA$ and less than $3.5 \AA$, such a distance is included in our instances as an interval of width $1 \AA$. All intervals have a predefined width and their extremes are randomly generated in a way that the interval contains the true distance.

\subsection{Numerical results}

Let us refer to the algorithm presented in [34] as $i \mathrm{BP}$, while we will name "New $i \mathrm{BP}$ " the algorithm integrated with the new method for the computation of candidate positions (see Sect. 2.3), with the technique for arc reduction (see Sect. 3), with the chirality and peptide plane pruning devices (see Sect. 2.2), and with the pruning device introduced in Sect. 4.3.

In both $i \mathrm{BP}$ variants, the tolerance used in the experiments for the DDF criterion (Eq. 3) is $\varepsilon=0.001$. In new $i \mathrm{BP}$, for the PMDE-based pruning device, we used $\hat{\varepsilon}=0.01$ and set $M=40$ in definition of $\mathcal{L}$ (see Eq. 7). We gradually increased the number of samples $D$ taken from the feasible arcs until the first solution is found in less than $60 \mathrm{~s}$ (timeout).

The numerical experiments were run in a Intel MacBook Pro, 2Ghz, 2GB RAM, and the Algorithm 1 was implemented in C programming language, compiled using GNU GCC with flag -O3.

Table 1 shows a comparison between $i \mathrm{BP}$ and New $i \mathrm{BP}$. The number of amino acids $(a a)$, atoms $(|V|)$ and available distances $(|E|)$ are given for each instance. For the two versions of $i \mathrm{BP}$, the performance is evaluated by the minimum number of samples $D$ (taken from interval arcs) necessary to find one solution, the number of recursive calls and the CPU time in seconds. The quality of the realization is assessed through the MDE. The character "**" means that the instance could not be solved in less than one minute for any value of $D \leq 20$.

We can notice that the arc reduction technique presented in Sect. 3 is very effective in reducing the number of sample positions that we need to extract from the arcs in order to obtain at least one solution. This is an important improvement because it is not known a priori how many samples are sufficient to allow $i \mathrm{BP}$ to find a conformation. We can observe that the number of calls and CPU time were reduced in 9 out of 11 instances. It is also worth to mention that the pruning criteria based on peptide plane geometry and chirality helped the new version of $i \mathrm{BP}$ in reducing the number of calls in some instances and improving the global fold as well.

Concerning the MDE, the original $i \mathrm{BP}$ seems to be more stable, although it fails to solve four of the instances (within the specified timeout). On the other hand, since the New 
Table 1 Numerical results on artificially generated instances from the PDB

\begin{tabular}{|c|c|c|c|c|c|c|c|c|c|c|c|}
\hline \multirow[t]{2}{*}{ PDB ID } & \multicolumn{3}{|c|}{ Instance } & \multicolumn{4}{|c|}{$i$ BP from $[34]$} & \multicolumn{4}{|c|}{ New $i$ BP } \\
\hline & $a a$ & $|V|$ & $|E|$ & $D$ & Calls & Time & MDE & $D$ & Calls & Time & MDE \\
\hline 2JMY & 15 & 120 & 660 & 13 & 37,658 & 0.13 & $3 e-06$ & 5 & 2983 & 0.01 & $1 e-16$ \\
\hline $2 \mathrm{KXA}$ & 24 & 177 & 973 & 10 & 215,669 & 0.92 & $5 e-06$ & 3 & 5064 & 0.01 & $6 e-03$ \\
\hline 1DSK & 28 & 222 & 1210 & 14 & 31,309 & 0.13 & $4 e-06$ & 4 & 53,890 & 0.14 & $1 e-06$ \\
\hline $2 \mathrm{PPZ}$ & 36 & 287 & 1522 & 9 & $2,372,242$ & 11.34 & $2 e-06$ & 3 & 442,965 & 1.87 & $4 e-08$ \\
\hline $1 \mathrm{AQR}$ & 40 & 310 & 1596 & $*$ & $*$ & $*$ & $*$ & 4 & 114,671 & 0.20 & $6 e-03$ \\
\hline 2ERL & 40 & 324 & 1792 & 14 & 1,4 & 6.1 & & 3 & 10,410 & 0.03 & $1 e-03$ \\
\hline $2 \mathrm{E} 2 \mathrm{~F}$ & 41 & 315 & 1716 & $*$ & $*$ & $*$ & $*$ & 3 & 19916 & 0.06 & $9 e-03$ \\
\hline 1FJK & 52 & 417 & 2306 & 12 & 115,426 & 0.73 & $4 e-06$ & 4 & 925,090 & 3.07 & $2 e-06$ \\
\hline $2 J W U$ & 56 & 448 & 2416 & $*$ & $*$ & $*$ & $*$ & 4 & 226870 & 0.81 & $1 e-02$ \\
\hline 2KIQ & 57 & 455 & 2452 & 20 & $1,217,945$ & 12.79 & $6 e-06$ & 4 & 317,136 & 1.12 & $7 e-04$ \\
\hline 2LOW & 64 & 497 & 2650 & $*$ & $*$ & $*$ & $*$ & 3 & $3,738,152$ & 8.79 & $2 e-07$ \\
\hline
\end{tabular}

$i$ BP uses the relaxed pruning criterion PMDE, it cannot ensure a better MDE for all the instances. For some of them we observe a better MDE which is a consequence of the other considered pruning devices. Although we relaxed some distance constraints by using PMDE, the chirality constraints helped in improving the local geometry, resulting in a better MDE. For those instances with a worse MDE, like 2KXA, 2E2F or 2KIQ, the PMDE relaxation was, in some sense, the way to "pass-through" the long-range distance constraints and find a realization in a affordable time. Additionally, we remark that an MDE value around $10^{-3}$ is able to guarantee a sufficient detection of the global fold of the protein. In fact, the realizations found by the New $i$ BP are not so far from the true ones. The quality of such realizations is discussed in the next subsection.

\subsection{Quality of a realization and practical usage}

While looking at Table 1, a natural question emerges: how good are the realizations $X$ with $M D E(X) \approx 10^{-3}$ when compared to the "true" protein ?

Since we have relaxed the distance constraints related to long-range distances, in principle, we cannot ensure that the underlying molecule is recovered. However, we will illustrate that the realization found by the New $i$ BP gives a very good approximation of the true conformation.

First, let us take a look at the instance 2KXA. Figure 7 shows the realizations found (first found solutions) by the original $i \mathrm{BP}$ and the New $i \mathrm{BP}$. Although the MDE of the first is smaller than the second, $10^{-6}$ against $10^{-3}$, we can see that the conformations are roughly the same, except by partial reflections. The New iBP produced a right-handed helix because it contains, in its list of pruning devices, the chirality-based device.

Now, let us consider the instance 2E2F. According to Table 1, the MDE for the realization found by the New $i \mathrm{BP}$ is approximately $10^{-2}$. By superimposing the realizations found with the true one from the PDB (first model), see Fig. 8, we obtain a RMSD value equal to $0.7 \AA$ (according to TM-align [67]).

Therefore, although the realization found by New $i$ BP does not fit perfectly with the true conformation, it is close enough to identify its global fold, and it also can be used as a smart 

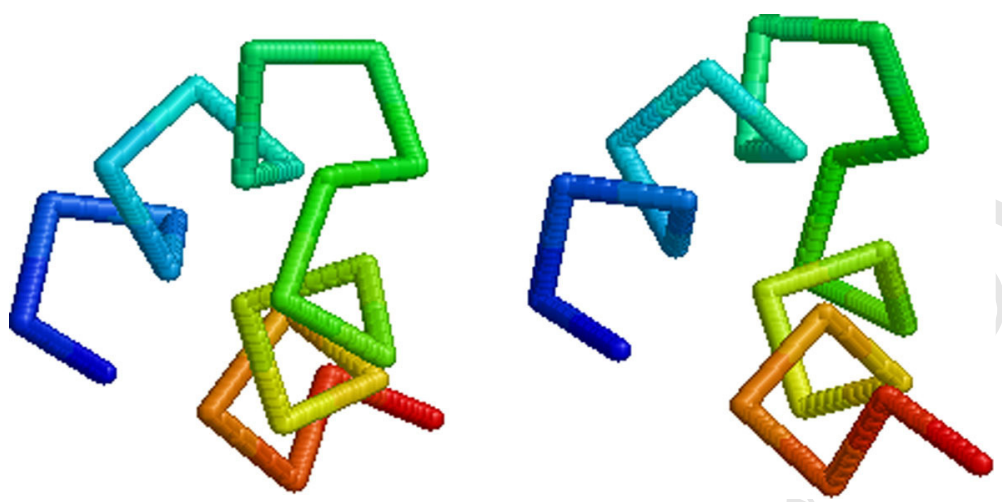

Fig. 7 Realization for $2 \mathrm{KXA}$ found by the original $i \mathrm{BP}($ left $)$ and the new $i \mathrm{BP}$ (right)

Fig. 8 Superimposition of solution found by the New $i$ BP (red) and the original PDB file (blue) for $2 \mathrm{E} 2 \mathrm{~F}$

starting point for a local optimization intended to minimize the MDE and enforce VdW constraints [61].

Once the first solution $X_{1}$ is found by the "New $i$ BP" , a set of feasible exact distances for the distances that were originally represented by intervals can be selected. This set of distances defines a DGP instance with exact distances which contains $X_{1}$ in its finite solution set. Moreover, by solving such an instance with the basic BP algorithm (for exact distances), we can compute all other feasible conformations that can be obtained from $X_{1}$ by partial reflections $[32,38]$. This procedure gets rid of the flexings ${ }^{1}$ in the molecule, but only in this case the solution set is finite.

Applying this scheme to a modified 1AQR instance, where hydrogen distances between consecutive amino-acids were removed and the threshold was lowered to $4.5 \AA$, four incongruent conformations are obtained, as depicted in Fig. 9.

We claim that, even though interval distances pose some difficulties to the extension of this combinatorial approach, it is still possible to explore all the (discrete) conformational space obtained with discretization. Henceforth, we propose our New $i$ BP as an exploratory tool to enumerate protein conformations that satisfy most of the given distance restraints, that can be further improved by local minimization procedures.

1 Continuous motions of part of the structure preserving all distance restraints. 

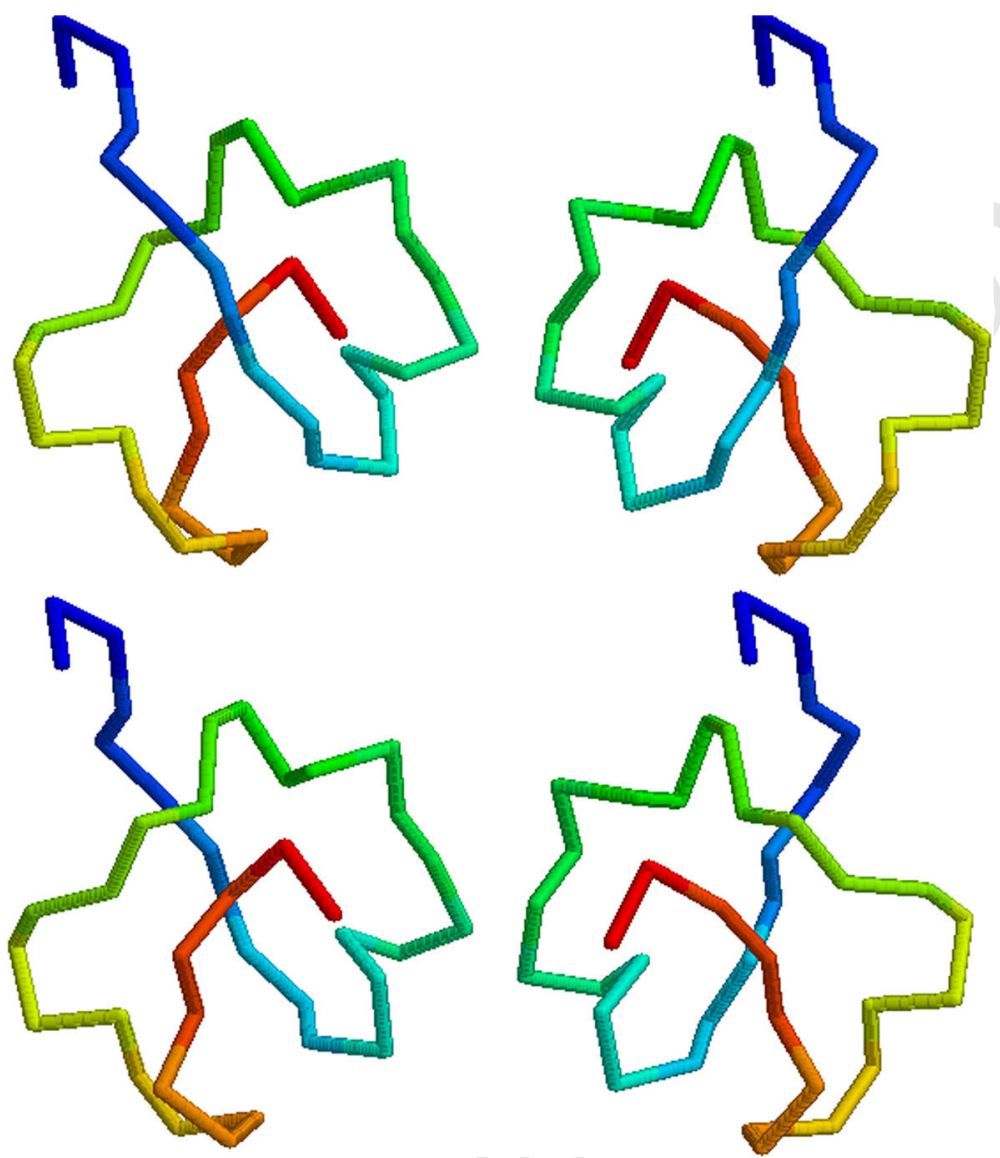

Fig. 9 Four incongruent realizations for 1AQR. The conformations in the bottom are reflections of the top ones. All four conformations differ by partial reflections

\section{Conclusion and future work}

We collected in this paper the most recent and promising advances in solving the MDGP with our combinatorial approach. The main contributions presented in this paper can be summarized as follows:

1. identification of the main barriers against the successful application of the discrete approach to real MDGPs with interval data;

2. another pruning devices based on chirality and peptide planes, whose easy implementation is allowed by the model and discretization order presented in Sect. 2.2;

3. a new pruning device that "relaxes" long-range distance constraints (Sect. 4.3) which allows us to obtain approximate realizations.

Computational experiments on artificially generated instances showed the effectiveness of all above mentioned points, when integrated in the $i \mathrm{BP}$ algorithm. We are in fact able to find approximate realizations for protein backbones up to 64 amino acids in an affordable time,

\section{를 Springer}


and with reasonable precision, that can be further improved by using our solutions as starting points for a local minimization solver.

In the presented experiments, the two compared versions of the $i \mathrm{BP}$ algorithm were both used for identifying only one solution to the problem. However, as remarked before and illustrated in Sect. 5.3, the $i \mathrm{BP}$ algorithm can potentially enumerate the entire solution set of a discretizable MDGP. Research is currently focused on efficiently enumerating all conformations belonging to the search tree. Due to the presence of interval distances, many solutions may belong to the same cluster/ensemble of conformations. Hence, the next step is to define a method to classify the solutions in equivalence classes and integrate $i \mathrm{BP}$ with an scheme able to pick only one representative conformation from each incongruent ensemble.

Acknowledgements DG and CL are thankful to the Brazilian research agencies FAPESP and CNPq for partial financial support. LL was partially supported by the "Bip:Bip" project within the ANR "Investissement d'Avenir" program. AM thanks University of Rennes 1 for financial support. AM and DG also acknowledge Brittany Region (France) for partial financial support.

\section{References}

1. Alfakih, A.Y., Khandani, A., Wolkowicz, H.: Solving Euclidean distance matrix completion problems via semidefinite programming. Comput. Optim. Appl. 12, 13-30 (1999)

2. Alipanahi, B., Krislock, N., Ghodsi, A., Wolkowicz, H., Donaldson, L., Li, M.: Determining protein structures from NOESY distance constraints by semidefinite programming. J. Comput. Biol. 20, 296-310 (2013)

3. Almeida, F.C.L., Moraes, A.H., Gomes-Neto, F.: An overview on protein structure determination by NMR: historical and future perspectives of the use of distance geometry methods. In: Mucherino et al. [52], pp. 377-412

4. Berman, H., Westbrook, J., Feng, Z., Gilliland, G., Bhat, T., Weissig, H., Shindyalov, I., Bourne, P.: The Protein Data Bank. Nucl. Acids Res. 28, 235-242 (2000)

5. Billeter, M., Braun, W., Wüthrich, K.: Sequential resonance assignments in protein ${ }^{1} \mathrm{H}$ nuclear magnetic resonance spectra. Computation of sterically allowed proton-proton distances and statistical analysis of proton-proton distances in single crystal protein conformations. J. Mol. Biol. 155, 321-346 (1982)

6. Biswas, P., Lian, T., Wang, T., Ye, Y.: Semidefinite programming based algorithms for sensor network localization. ACM Trans. Sens. Netw. 2, 188-220 (2006)

7. Bizien, T., Durand, D., Roblina, P., Thureau, A., Vachette, P., Péreza, J.: A brief Survey of State-of-the-Art BioSAXS. Protein Pept. Lett. 23, 217-231 (2016)

8. Cassioli, A., Bordeaux, B., Bouvier, G., Mucherino, A., Alves, R., Liberti, L., Nilges, M., Lavor, C., Malliavin, T.: An algorithm to enumerate all possible protein conformations verifying a set of distance constraints. BMC Bioinform. 16, 16-23 (2015)

9. Cassioli, A., Gunluk, O., Lavor, C., Liberti, L.: Discretization vertex orders in distance geometry. Discrete Appl. Math. 197, 27-41 (2015)

10. Chen, Z.A., Jawhari, A., Fischer, L., Buchen, C., Tahir, S., Kamenski, T., Rasmussen, M., Lariviere, L., Bukowski-Wills, J.-C., Nilges, M., Cramer, P., Rappsilber, J.: Architecture of the RNA polymerase II-TFIIF complex revealed by cross-linking and mass spectrometry. EMBO J. 29, 717-726 (2010)

11. Costa, V., Mucherino, A., Lavor, C., Cassioli, A., Carvalho, L.M., Maculan, N.: Discretization orders for protein side chains. J. Glob. Optim. 60, 333-349 (2014)

12. Crippen, G., Havel, T.: Distance Geometry and Molecular Conformation. Wiley, New York (1988)

13. Dattorro, J.: Convex Optimization and Euclidean Distance Geometry. $\mathcal{M} \epsilon \beta$ oo, Palo Alto (2005)

14. Dokmanic, I., Parhizkar, R., Ranieri, J., Vetterli, M.: Euclidean distance matrices: essential theory, algorithms, and applications. Sig. Process. Mag. IEEE 32(6), 12-30 (2015)

15. Dong, Q., Wu, Z.: A linear-time algorithm for solving the molecular distance geometry problem with exact inter-atomic distances. J. Glob. Optim. 22, 365-375 (2002)

16. Dong, Q., Wu, Z.: A geometric build-up algorithm for solving the molecular distance geometry problem with sparse distance data. J. Glob. Optim. 26(3), 321-333 (2003). doi:10.1023/A:1023221624213

17. Ferguson, D., Marsh, A., Metzger, T., Garrett, D., Kastella, K.: Conformational searches for the global minimum of protein models. J. Glob. Optim. 4, 209-227 (1994) 
18. Fiorioto, F., Damberger, F., Herrmann, T., Wüthrich, K.: Automated amino acid side-chain NMR assignment of proteins using 13C- and 15N-resolved 3D [1H,1H]-NOESY. J. Biomol. NMR 42, 23-33 (2008)

19. Gonçalves, D.S., Mucherino, A.: Discretization orders and efficient computation of cartesian coordinates for distance geometry. Optim. Lett. 8, 2111-2125 (2014)

20. Gonçalves, D.S., Mucherino, A., Lavor, C.: An adaptive branching scheme for the branch \& prune algorithm applied to distance geometry. In: IEEE Conference Proceedings, pp. 463-469. Workshop on Computational Optimization (WCO14), FedCSIS14, Warsaw, Poland (2014)

21. Grand, S.L., Merz, K.: The application of the genetic algorithm to the minimization of potential energy functions. J. Glob. Optim. 3, 49-66 (1993)

22. Guerry, P., Duong, V.D., Herrmann, T.: CASD-NMR 2: robust and accurate unsupervised analysis of raw NOESY spectra and protein structure determination with UNIO. J. Biomol. NMR 62, 473-480 (2015)

23. Güntert, P.: Automated NMR structure calculation with CYANA. Methods Mol. Biol. 278, 353-378 (2004)

24. Herrmann, T., Güntert, P., Wüthrich, K.: Protein NMR structure determination with automated NOE assignment using the new software CANDID and the torsion angle dynamics algorithm DYANA. J. Mol. Biol. 319, 209-227 (2002)

25. Herrmann, T., Güntert, P., Wüthrich, K.: Protein NMR structure determination with automated NOEidentification in the NOESY spectra using the new software ATNOS. J. Biomol. NMR 24, 171-189 (2002)

26. L, Hoai An: Solving large scale molecular distance geometry problems by a smoothing technique via the Gaussian transform and d.c. programming. J. Glob. Optim. 27, 375-397 (2003)

27. Huang, H.X., Liang, Z.A., Pardalos, P.: Some properties for the Euclidean distance matrix and positive semidefinite matrix completion problems. J. Glob. Optim. 25, 3-21 (2003)

28. Kirkpatrick, S., Gelatt, C.D., Vecchi, M.P.: Optimization by simulated annealing. Science 220, 671-680 (1983)

29. Krislock, N., Wolkowicz, H.: Explicit sensor network localization using semidefinite representations and facial reductions. SIAM J. Optim. 20, 2679-2708 (2010)

30. Lavor, C., Alves, R., Figueiredo, W., Petraglia, A., Maculan, N.: Clifford algebra and the discretizable molecular distance geometry problem. Adv. Appl. Clifford Algebr. 25, 925-942 (2015)

31. Lavor, C., Lee, J., John, A.L.S., Liberti, L., Mucherino, A., Sviridenko, M.: Discretization orders for distance geometry problems. Optim. Lett. 6, 783-796 (2012)

32. Lavor, C., Liberti, L., Maculan, N., Mucherino, A.: The discretizable molecular distance geometry problem. Comput. Optim. Appl. 52, 115-146 (2012)

33. Lavor, C., Liberti, L., Maculan, N., Mucherino, A.: Recent advances on the discretizable molecular distance geometry problem. Eur. J. Oper. Res. 219, 698-706 (2012)

34. Lavor, C., Liberti, L., Mucherino, A.: The interval branch-and-prune algorithm for the discretizable molecular distance geometry problem with inexact distances. J. Glob. Optim. 56, 855-871 (2013)

35. Lavor, C., Mucherino, A., Liberti, L., Maculan, N.: On the computation of protein backbones by using artificial backbones of hydrogens. J. Glob. Optim. 50, 329-344 (2011)

36. Liberti, L., Lavor, C., Maculan, N.: A branch-and-prune algorithm for the molecular distance geometry problem. Int. Trans. Oper. Res. 15, 1-17 (2008)

37. Liberti, L., Lavor, C., Maculan, N., Marinelli, F.: Double variable neighbourhood search with smoothing for the molecular distance geometry problem. J. Glob. Optim. 43, 207-218 (2009)

38. Liberti, L., Lavor, C., Maculan, N., Mucherino, A.: Euclidean distance geometry and applications. SIAM Rev. 56, 3-69 (2014)

39. Liberti, L., Lavor, C., Mucherino, A., Maculan, N.: Molecular Distance Geometry Methods: from Continuous to Discrete. Int. Trans. Oper. Res. 18, 33-51 (2011)

40. Liberti, L., Lavor, C., Mucherino, A.: The discretizable molecular distance geometry problem seems easier on proteins. In: Mucherino, A., Lavor, C., Liberti, L., Maculan, N. (eds.) Distance Geometry, pp. 47-60. Springer, New York (2013)

41. Liberti, L., Masson, B., Lee, J., Lavor, C., Mucherino, A.: On the number of realizations of certain Henneberg graphs arising in protein conformation. Discrete Appl. Math. 165, 213-232 (2014)

42. Linge, J.P., Habeck, M., Rieping, W., Nilges, M.: ARIA: automated NOE assignment and NMR structure calculation. Bioinformatics 19, 315-316 (2003)

43. Locatelli, M., Schoen, F.: Minimal interatomic distance in morse clusters. J. Glob. Optim. 22(1), 175-190 (2002). doi:10.1023/A:1013811230753

44. Malliavin, T., Mucherino, A., Nilges, M.: Distance geometry in structural biology: new perspectives. In: Mucherino et al. [52], pp. 329-350

45. Man-Cho So, A., Ye, Y.: Theory of semidefinite programming for sensor network localization. Math. Program. B 109, 367-384 (2007) 
46. Maranas, C., Floudas, C.: Global minimum potential energy conformations of small molecules. J. Glob. Optim. 4, 135-170 (1994)

47. Moré, J., Wu, Z.: Distance geometry optimization for protein structures. J. Glob. Optim. 15(3), 219-234 (1999). doi:10.1023/A:1008380219900

48. Moré, J., Wu, Z.: Distance geometry optimization for protein structures. J. Glob. Optim. 15, 219-223 (1999)

49. Mucherino, A.: On the identification of discretization orders for distance geometry with intervals. In: Proceedings of Geometric Science of Information (GSI13), pp. 231-238. Lecture Notes in Computer Science 8085, Paris, France (2013)

50. Mucherino, A.: A pseudo De Bruijn graph representation for discretization orders for distance geometry. In: Proceedings of the 3rd International Work-Conference on Bioinformatics and Biomedical Engineering (IWBBIO15), Part I, Lecture Notes in Bioinformatics, vol. 9043, pp. 514-523. Granada, Spain (2015)

51. Mucherino, A., Lavor, C., Liberti, L.: The discretizable distance geometry problem. Optim. Lett. 6, $1671-1686(2012)$

52. Mucherino, A., Lavor, C., Liberti, L., Maculan, N. (eds.): Distance Geometry: Theory, Methods and Applications. Springer, New York (2013)

53. Mucherino, A., Lavor, C., Malliavin, T., Liberti, L., Nilges, M., Maculan, N.: Influence of pruning devices on the solution of molecular distance geometry problems. In: Pardalos, P.M., Rebennack, S. (eds.) Proceedings of the 10th International Symposium on Experimental Algorithms (SEA11), Lecture Notes in Computer Science, vol. 6630, pp. 206-217. Crete, Greece (2011)

54. Ryu, J., Kim, D.S.: Protein structure optimization by side-chain positioning via beta-complex. J. Glob. Optim. 57(1), 217-250 (2013). doi:10.1007/s10898-012-9886-3

55. Santana, R., Larrañaga, P., Lozano, J.: Side chain placement using estimation of distribution algorithms. Artif. Intell. Med. 39, 49-63 (2007)

56. Saxe, J.B.: Embeddability of weighted graphs in $k$-space is strongly NP-hard. In: Proceedings of 17 th Allerton Conference in Communications. Control and Computing, pp. 480-489. Monticello, IL (1979)

57. Schlick, T.: Molecular Modelling and Simulation: An Interdisciplinary Guide. Springer, New York (2002)

58. Schoenberg, I.: Remarks to Maurice Fréchet's article "Sur la définition axiomatique d'une classe d'espaces distanciés vectoriellement applicable sur l'espace de Hilbert”. Ann. Math. 36, 724-732 (1935)

59. Sippl, M., Scheraga, H.: Cayley-Menger coordinates. Proc. Natl. Acad. Sci. USA 83, 2283-2287 (1986)

60. Sit, A., Wu, Z.: Solving a generalized distance geometry problem for protein structure determination. Bull. Math. Biol. 73, 2809-2836 (2011)

61. Souza, M., Lavor, C., Muritiba, A., Maculan, N.: Solving the molecular distance geometry problem with innacurate distance data. BMC Bioinform. 14((Suppl. 9):S7), 1-6 (2013)

62. Thompson, H.: Calculation of cartesian coordinates and their derivatives from internal molecular coordinates. J. Chem. Phys. 47, 3407-3410 (1967)

63. Volk, J., Herrmann, T., Wüthrich, K.: Automated sequence-specific protein NMR assigment using memetic algorithm MATCH. J. Biomol. NMR 41, 127-138 (2008)

64. Wu, D., Wu, Z.: An updated geometric build-up algorithm for solving the molecular distance geometry problem with sparse distance data. J. Glob. Optim. 37, 661-673 (2007)

65. Wu, D., Wu, Z., Yuan, Y.: Rigid versus unique determination of protein structures with geometric buildup. Optim. Lett. 2, 319-331 (2008)

66. Wüthrich, K., Billeter, M., Braun, W.: Pseudo-structures for the 20 common amino acids for use in studies of protein conformations by measurements of intramolecular proton-proton distance constraints with Nuclear Magnetic Ressonance. J. Mol. Biol. 169, 949-961 (1983)

67. Zhang, Y., Skolnick, J.: TM-align: a protein structure alignment algorithm based on TM-score. Nucl. Acids Res. 33, 2302-2309 (2005)

68. Zou, Z., Bird, R., Schnabel, R.: A stochastic/perturbation global optimization algorithm for distance geometry problems. J. Glob. Optim. 11(1), 91-105 (1997). doi:10.1023/A:1008244930007

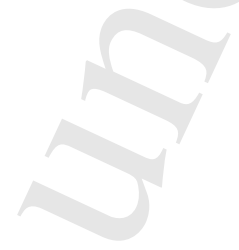

